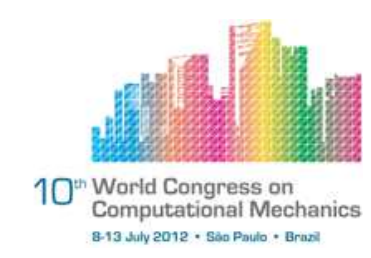

\title{
FINITE ELEMENT APPLICATION TO DUCTILE FRACTURE PROBLEMS INVOLVING HIGH NUMBER OF DEGREES OF FREEDOM
}

\author{
Koffi Enakoutsa
}

Eutaw Construction Company, LLC, Starkville, MS (enakoutsa@yahoo.fr)

\begin{abstract}
The aim of this paper is to show, using an example, the finite element potential to simulate ductile fracture problems involving high number of degrees of freedom. The example consists of a model proposed by Gologanu, Leblond, Perrin and Devaux (GLPD model) to describe ductile fracture. This model is an extension of the famous Gurson's model to address the underlying unlimited localization problem arising in the Gurson model. The GLPD model was derived from some refinement of Gurson's original homogenization procedure; the new model is of "micromorphic" nature, involving the second gradient of the macroscopic velocity and generalized macroscopic stresses of "moment" type, together with some characteristic "microstructural distance". The numerical implementation of this model into finite element codes is quite involved, since its requires the use of finite element of class $C^{1}$ and the solution of a complex "projection onto the yield locus" problem. Enakoutsa and Leblond have proposed a numerical scheme that avoids these two difficulties. We present here some new assessments of this numerical scheme. First, we develop an analytical solution for the problem of an elastic hollow sphere, obeying the GLPD model and subjected to hydrostatic tension; this solution agrees very well with the numerical predictions of the GLPD model. Also, comparisons between experimental and numerical load vs. displacement curves for an axisymmetric pre-cracked spcimen made of typical stainless steel are found to yield satisfatory results.
\end{abstract}

Keywords: Ductile Fracture, Micromorphic, Numerical implementation. 


\section{INTRODUCTION}

Ductile fracture of porous metals arises from the nucleation, growth and final coalescence of microvoids. Gurson (1977)'s model based on a "homogenized" approach provides a good description of the second stage of this process. Heuristic extensions of Gurson model due to Tvergaard (1981) and Tvergaard and Needleman (1984) allow to account for the first and third stages. Practical finite element computations of problems involving ductile fracture always suffer from a pathological dependency of the results upon the finite element size. This problem originates from the presence of softening in the Gurson model. One way to circumvate this difficulty is to adopt a nonlocal evolution equation for the porosity (softening parameter) involving a spatial integral of some "local porosity rate", as suggested by Pijaudier-Cabot and Bazant (1987) in the context of concrete damage. Enakoutsa et al. (2007) have shown that this solution allows to eliminate the pathological mesh effects without degrading the quality of the agreement between typical experiments of ductile fracture tests. However, the succes of this approach was at the expense of its purely heuristic character and its lack of any serious physical justification. Boundary effects also entail some drawbacks.

These are good reasons to consider Gologanu et al. (1997)'s micromorphic model of ductile rupture, which was derived from some extension of Gurson (1977)'s original homogenization procedure, based on conditions of homogeneous boundary strain rate, to conditions of inhomogeneous boundaries strain rate. In Gologanu et al. (1997) model, the velocity imposed on the boundary of the representative cell considered is no longer linear but quadratic with respect to the coordinates. Doing so allows to account for sharp gradient of macroscopic mechanical fields encountered, for instance, near crack tips or during strain localization. The output of the procedure was a model of "micromorphic" type, involving the second gradient of the macroscopic velocity and a generalized macroscopic stress of "moment" type together with some microstructural characteristic distance of the order of the average voids semi-spacing.

The numerical implementation of this model into some finite element code raises two problems. The first one is the apparent need for finite element of class $C^{1}$, non-available in standard finite element codes. This need is obviated through introduction of some new nodal variables representing the components of the strain rate. The second difficulty lies in the necessary operation of "projec- 
tion" onto the sophisticated yield locus. An implicit algorithm similar in principle to that classically used for the von Mises criterion, although much more complex in detail, is adopted for this purpose. The details of the numerical implementation are provided in Enakoutsa and Leblond (2009).

The aim of this paper is to follow up the study of the assessement of the algorithm of Enakoutsa and Leblond. Namely, we shall consider two different assessments. The first one is discussed in Section 2. It is concerned with some analytic solution developed for the problem of an elastic hollow sphere subjected to hydrostatic tension in the framework of linearized elasticity; the matrix material obeys the GLPD model. Comparisions between the numerical predictions of the GLPD model and the analytical solution confirm the robustness of the numerical scheme used by Enakoutsa and Leblond (2009) in the implementation of this model into SYSTUS finite element code. Hence, this reference analytical solution can be used to assess the numerical implementation of the GLPD model in another finite element code. In the second application presented in Section 3, comparisions between experimental and numerical load-displacement curves for an axisymmetric pre-cracked specimen made of a typical stainless steel are found to yield satisfactory results.

\section{GLPD model}

The derivation of the GLPD model, based on homogenization of some representative "elementary cell" in some plastic porous medium subjected to conditions of inhomogeneous boundary strain rate, is presented in detail in Gologanu et al. (1997) and will not be repeated here. The hypothesis of additivity of elastic and plastic strain rates reads

$$
\left\{\begin{array}{l}
\mathbf{D} \equiv \mathbf{D}^{e}+\mathbf{D}^{p} \\
\nabla \mathbf{D} \equiv(\nabla \mathbf{D})^{e}+(\nabla \mathbf{D})^{p} .
\end{array}\right.
$$

Note that the elastic and plastic parts $(\nabla \mathbf{D})^{e},(\nabla \mathbf{D})^{p}$ of the gradient of the strain rate here have no reason to coincide with the gradients $\nabla\left(\mathbf{D}^{e}\right), \nabla\left(\mathbf{D}^{p}\right)$ of the elastic and plastic parts of the strain rate. The rest of the elements of the constitutive equations of the GLPD model are summarized below. 


\subsection{Hypoelasticity law}

The elastic parts of the strain rate and its gradient are related to the rates of the stresses and moments through the following hypoelasticity law ${ }^{1}$ :

$$
\left\{\begin{array}{l}
\check{\sigma}_{i j}=\lambda D_{k k}^{e} \delta_{i j}+2 \mu D_{i j}^{e} \\
\check{M}_{i j k}=\frac{b^{2}}{5}\left[\lambda(\nabla D)_{k k}^{e} \delta_{i j}+2 \mu(\nabla D)_{i j}^{e}-2 \lambda U_{k}^{e} \delta_{i j}-2 \mu\left(U_{i}^{e} \delta_{j k}+U_{j}^{e} \delta_{i k}\right)\right] .
\end{array}\right.
$$

In these expressions $\check{\sigma}_{i j}$ and $\check{M}_{i j k}$ are the Jaumann (objective) derivatives of $\sigma_{i j}$ and $M_{i j k}$. Also, $\lambda$ and $\mu$ are Lamé's coefficients, $b$ is the mean half-spacing between voids ${ }^{2}$, and $\mathbf{U}^{e} \equiv\left(U_{i}^{e}\right)_{1 \leq i \leq 3}$ is a vector the value of which is fixed by the equations $\check{M}_{i j j}=0$ :

$$
U_{i}^{e}=\frac{\lambda(\nabla D)_{h h i}^{e}+2 \mu(\nabla D)_{i h h}^{e}}{2 \lambda+8 \mu} .
$$

This vector plays the same role in the model as the component $\epsilon_{z z}^{e}$ of the elastic strain in the theory of thin plates, which is free a priori but fixed in fine by the plane stress condition.

\subsection{Yield criterion}

The plastic behaviour is governed by the following Gurson-like criterion

$$
\Phi(\sigma, \mathbf{M}, f) \equiv \frac{1}{\bar{\sigma}^{2}}\left(\sigma_{e q}^{2}+\frac{Q^{2}}{b^{2}}\right)+2 p \cosh \left(\frac{3}{2} \frac{\sigma_{m}}{\bar{\sigma}}\right)-1-p^{2}=0 .
$$

In this expression,

- $\sigma_{e q} \equiv\left(\frac{3}{2} \sigma_{i j}^{\prime} \sigma_{i j}^{\prime}\right)^{1 / 2}\left(\sigma^{\prime} \equiv\right.$ deviator of $\left.\sigma\right)$ is the von Mises equivalent stress;

- $\sigma_{m} \equiv \frac{1}{3} \operatorname{tr} \sigma$ is the mean stress;

- $\bar{\sigma}$ represents a kind of average value of the yield stress in the heterogeneous metallic matrix, the evolution equation of which is given below;

${ }^{1}$ This law slightly differs from that proposed by Gologanu et al. (1997). The modification is permissible in view of the minor role played by elasticity in ductile rupture problems and allows for a somewhat easier numerical implementation of the model.

${ }^{2}$ This is the radius of the spherical elementary cell considered in the homogenization procedure. 
- $p$ is a parameter connected to the porosity (void volume fraction) $f$ through the relation (Tvergaard (1981), Tvergaard and Needleman (1984)):

$$
p \equiv q f^{*} \quad, \quad f^{*} \equiv \begin{cases}f & \text { if } f \leq f_{c} \\ f_{c}+\delta\left(f-f_{c}\right) & \text { if } f>f_{c}\end{cases}
$$

where $q$ is "Tvergaard's parameter", $f_{c}$ the "critical" porosity at the onset of coalescence of voids, and $\delta(>1)$ a factor describing the accelerated degradation of the material during coalescence;

- $Q^{2}$ is a quadratic form of the components of the moment tensor given by

$$
Q^{2} \equiv A_{I} M_{I}+A_{I I} M_{I I} \quad, \quad\left\{\begin{array}{l}
A_{I}=0.194 \\
A_{I I}=6.108
\end{array}\right.
$$

where $M_{I}$ and $M_{I I}$ are the first two invariants of $\mathbf{M}$ :

$$
M_{I} \equiv \frac{1}{9} M_{j j i} M_{k k i} \quad, \quad M_{I I} \equiv \frac{3}{2} M_{i j k}^{\prime} M_{i j k}^{\prime}
$$

$\left(\mathrm{M}^{\prime} \equiv\right.$ deviator of $\mathrm{M}$ over its first two indices).

\subsection{Flow rule}

The plastic parts of the strain rate and its gradient are given by the flow rule associated to the criterion (4) via the normality property ${ }^{3}$ :

$$
\left\{\begin{array}{l}
D_{i j}^{p}=\eta \frac{\partial \Phi}{\partial \sigma_{i j}} \\
(\nabla D)_{i j k}^{p}=\eta \frac{\partial \Phi}{\partial M_{i j k}}+U_{i}^{p} \delta_{j k}+U_{j}^{p} \delta_{i k}
\end{array}\right.
$$

with

$$
\left\{\begin{array}{l}
\eta=0 \text { if } \Phi(\sigma, \mathbf{M}, f)<0 \\
\eta \geq 0 \text { if } \Phi(\sigma, \mathbf{M}, f)=0 .
\end{array}\right.
$$

The terms involving the vector $\mathbf{U}^{p} \equiv\left(U_{i}^{p}\right)_{1 \leq i \leq 3}$ here represent an arbitrary rigidbody motion left unspecified by the flow rule (see Gologanu et al. (1997)). In practice, the value of $\mathbf{U}^{p}$ is again fixed by conditions $\check{M}_{i j j}=0$. This vector plays the same role in the model as the component $\dot{\epsilon}_{z z}^{p}$ of the plastic strain rate in the theory of thin plates.

\footnotetext{
${ }^{3}$ It has been shown by Gologanu et al. (1997) that the normality property is preserved in the homogenization process.
} 


\subsection{Evolution of internal parameters}

The evolution of the porosity is governed by the following classical equation, which results from approximate incompressibility of the metallic matrix:

$$
\dot{f}=(1-f) \operatorname{tr} \mathbf{D}^{p} \text {. }
$$

The parameter $\bar{\sigma}$ is given by

$$
\bar{\sigma} \equiv \sigma(\bar{\epsilon})
$$

where $\sigma(\epsilon)$ is the function providing the yield stress of the matrix material as a function of the equivalent cumulated strain $\epsilon$, and $\bar{\epsilon}$ is the average value of this equivalent strain in the heterogeneous matrix. The evolution of $\bar{\epsilon}$ is governed by the following equation:

$$
(1-f) \bar{\sigma} \dot{\bar{\epsilon}}=\sigma: \mathbf{D}^{p}+\mathbf{M}:(\nabla \mathbf{D})^{p}
$$

\section{Analytical solution of the hollow sphere problem}

In this section, we consider the problem of a hollow sphere of inner and outer radii $r_{i}$ and $r_{e}$, respectively. The inner surface of this sphere is fixed while the outer one is subjected to hydrostatic tension loading. The matrix material of th ehollow sphere obeys the elasticity law Eq.(2). We study spherically symmetric solutions for this problem in the framework of linearized elasticity (small displacement, small strain).

Let $u_{r} \equiv u$ denote the radial displacement in the matrix, $\mathbf{u} \equiv u \mathbf{e}_{r}$ the displacement vector; also, we assume that $\mathbf{w}=\Delta \mathbf{u}$. Taking the derivatives of the moment and stress components of Eq.(2), we get after a tedious calculation

$$
\left\{\begin{array}{l}
\sigma_{i j, j}=(\lambda+2 \mu) w_{i} \\
M_{i j k, j k}=\mu \frac{10}{b^{2}} \frac{\lambda+2 \mu}{\lambda+4 \mu}(\Delta w)_{i} .
\end{array}\right.
$$

The equilibrium equations $\sigma_{i j, j}-M_{i j k, j k}=0$ yields the following equation:

$$
\Delta \mathbf{w}-k^{2} \mathbf{w}=0,
$$

where

$$
k^{2}=\frac{1-2 \nu}{2-3 \nu} \frac{b}{5} .
$$


Eq.(14) is similar to those obtained in the problem of radial vabriations of a sphere, but with $-k^{2} \mathbf{w}$ instead of $k^{2} \mathbf{w}$. In fact, it can be shown that the vector $\mathbf{w}$ derives from a function $\Phi$, that is, $\mathbf{w}=\operatorname{grad} \Phi$. Eq.(14) then yields

$$
\Delta \Phi-k^{2} \Phi=\text { cste }
$$

Adjusting the arbitrary cste in Eq.(16) and solving this equation for $\Phi$ we get in spherical coordinates

$$
\Phi=\alpha \frac{\mathrm{e}^{k r}}{r}+\beta \frac{\mathrm{e}^{-k r}}{r} \quad \text { and } \quad w \equiv w_{r}=\Phi^{\prime} .
$$

where $\alpha, \beta$ and $k$ are arbitrary constants and the symbol ' denotes $\frac{\partial}{\partial r}$. The radial displacement $u_{r} \equiv u$ is then obtained as

$$
w \equiv w_{r}=(\nabla \operatorname{tr} \mathbf{D})_{, r}=(\operatorname{tr} \mathbf{D})_{, r}=\left(u^{\prime}+\frac{2 u}{r}\right)^{\prime} .
$$

Solving Eq.(18) for $u_{r} \equiv u$, we finally get

$$
u(x)=\alpha\left(\frac{1}{x}-\frac{1}{x^{2}}\right) \mathrm{e}^{x}+\beta\left(\frac{1}{x}+\frac{1}{x^{2}}\right) \mathrm{e}^{-x}+\gamma x+\frac{\delta}{x^{2}},
$$

where $x \equiv k r, \alpha, \beta, \gamma$ and $\delta$ are arbitrary constants. It is necessary to calcultate the values of these arbitrary constants to completly define the analytical form of the displacement $u$. To that end, we define the boundary conditions $M_{r r r}\left(r_{i}\right)=0$, $u_{r}\left(r_{i}\right)=\Delta_{i}, M_{r r r}\left(r_{e}\right)=0$ and $u_{r}\left(r_{e}\right)=\Delta_{e}$. This allows to form a system of four linear equations with four unknowns $\alpha, \beta, \gamma$ and $\delta$

$$
\left\{\begin{array}{l}
\alpha \mathrm{e}^{x_{i}} P_{i}+\beta \mathrm{e}^{-x_{i}} Q_{i}+\frac{6 \delta}{x_{i}^{4}}=0 \\
\alpha \mathrm{e}^{x_{e}} P_{e}+\beta \mathrm{e}^{-x_{e}} Q_{e}+\frac{6 \delta}{x_{e}^{4}}=0 \\
\alpha\left(\frac{1}{x_{i}}+\frac{1}{x_{i}^{2}}\right) \mathrm{e}^{x_{i}}-\beta\left(\frac{1}{x_{i}}+\frac{1}{x_{i}^{2}}\right) \mathrm{e}^{-x_{i}}+\gamma x_{i}+\frac{\delta}{x_{i}^{2}}=\Delta_{i} \\
\alpha\left(\frac{1}{x_{e}}+\frac{1}{x_{e}^{2}}\right) \mathrm{e}^{x_{e}}-\beta\left(\frac{1}{x_{e}}+\frac{1}{x_{e}^{2}}\right) \mathrm{e}^{-x_{e}}+\gamma x_{e}+\frac{\delta}{x_{e}^{2}}=\Delta_{e}
\end{array}\right.
$$


the solution of which is obtained as:

$$
\left\{\begin{array}{l}
\alpha=\frac{6\left(\frac{\Delta_{i}}{x_{i}}-\frac{\Delta_{e}}{x_{e}}\right)\left(x_{i}^{4} Q_{i} \mathrm{e}^{-x_{i}}-x_{e}^{4} Q_{e} \mathrm{e}^{-x_{e}}\right)}{\mathcal{D}_{e}} \\
\beta=-\frac{6\left(\frac{\Delta_{i}}{x_{i}}-\frac{\Delta_{e}}{x_{e}}\right)\left(x_{i}^{4} P_{i} \mathrm{e}^{x_{i}}-x_{e}^{4} P_{e} \mathrm{e}^{x_{e}}\right)}{\mathcal{D}_{e}} \\
\delta=-\frac{\alpha x_{i}^{4} \mathrm{e}^{x_{i}} P_{i}+\beta x_{i}^{4} \mathrm{e}^{-x_{i}} Q_{i}}{6} \\
\gamma=\frac{\Delta_{i}}{x_{i}}-\left[\alpha\left(\frac{1}{x_{i}^{2}}-\frac{1}{x_{i}^{3}}\right) \mathrm{e}^{x_{i}}-\beta\left(\frac{1}{x_{i}^{2}}+\frac{1}{x_{i}^{3}}\right) \mathrm{e}^{-x_{i}}-\frac{\delta}{x_{i}^{3}}\right],
\end{array}\right.
$$

where $x_{i}=k r_{i}, x_{e}=k r_{e}, P_{i} \equiv P\left(x_{i}\right), P_{e} \equiv P\left(x_{e}\right), Q_{i} \equiv Q\left(x_{i}\right)$,

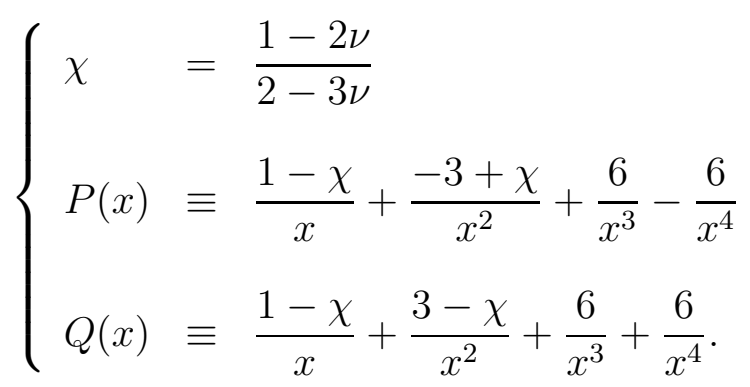

Eqs.(21), (19), (22) and the expression of $\mathcal{D}_{e}{ }^{4}$ completly define the expression of the radial displacement.

\section{Numerical applications}

Taking advantage of the axisymmetry conditions of the problem considered, we study only the quarter of the geometry of the problem, modeled in two dimensions; this yields a mesh discretization of 81 elements. The external radius $r_{e}$ worth $10 \mathrm{~mm}$ and is twice the internal radius $r_{i}$. Again, the matrix material of the hollow sphere obeys the elasticity law defined by Eq.(2), but using a high value of the yield limit, so that the behavior of the matrix stays in the elastic regime during the entire numerical computation. The value chosen for the characteristic length is $b=1 \mathrm{~mm}$. The load is an imposed displacement on the upper surface of

\footnotetext{
${ }^{4}$ The terms giving $\mathcal{D}_{e}$ are to long and are not provided here; however, it can be easily calculatedvusing Eq.(20)
} 
the hollow sphere; the inner surface of the sphere remains fix.

The numerical computations were performed using SYSTUS FE code developed by ESI Group where the GLPD model presented in Section 1 was implemented, in two dimensions. The numerical implementation has been extensively discussed in Enakoutsa and Leblond (2009); consequently, it will not be repeated here. Recall, however, that this implementation raises two majors problems. The first one is the apparent need for elements of class $C^{1}$. This need is obviated through introduction of some new nodal variables representing the components of the strain rate. The second problem lies in the necessary operation of "projection" onto the sophisticated yield locus. An implicit algorithm similar in principle to that classically used for the von Mises criterion, although much more complex in detail,is adopted for this purpose. The convergence of this algorithm is difficult, because of the large number of nodal degrees of freedom that it involves, and the CPU time may increase.

In practise, Enakoutsa and Leblond (2009) have obtained converged results by slightly modifying the original algorithm. Their modification consists of fixing the values of the rate of the plastic deformation as well as its gradient to those of the previous time step. These values become knowns in the problem, contrary to the original algorithm where they were unknowns. The solution of the new problem is then equivalent to that of a purely elastic problem with initial deformations. The balance equations are solved for on the configuration at time $t$ instead of $t+\Delta t$ and it only remains some weak non-linearities related to the Jaumann derivative of the stresses and moments. At convergence, the values of the rate of the plastic deformation and its gradient are stored, and then distributed into elastic and plastic parts using the "projection algorithm". The projection problem itself does not change. The proposed modification was only valid for very small time step as it is usually the case in explicit numerical codes.

Presented in contrast here are the results using the original implementation algorithm described in Enakoutsa and Leblond (2009). Figure1 illustrate the distribution of the displacement over a radius of the hollow sphere for several characteristic length distances $b$ : $0.5,1$ and $2 \mathrm{~mm}$. The results of these comparisons confirm the robustness of the numerical scheme proposed by Enakoutsa and Leblond (2009) to implement the GLPD model into SYSTUS FE code. Also, they demonstrate that the analytical solution for problem of an elastic hollow sphere obeying 


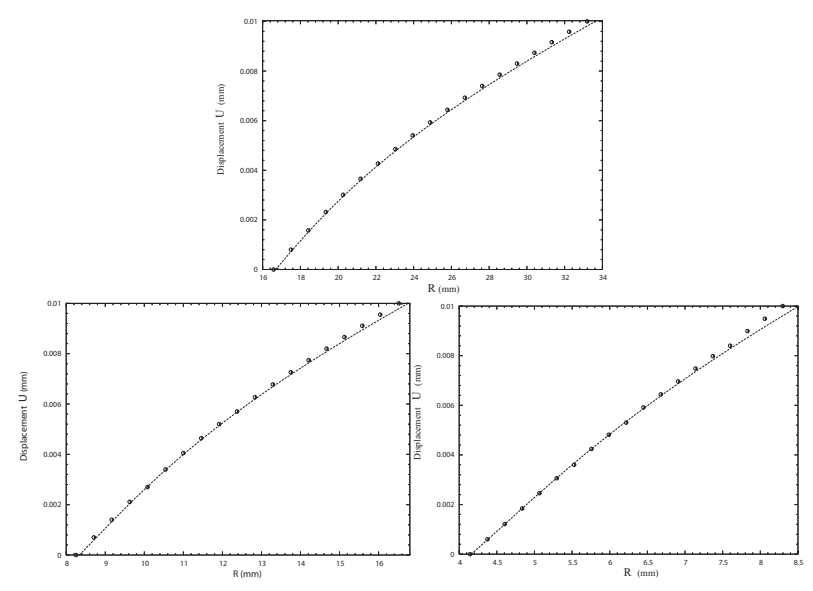

Figure 1: Comparisons computations/theory. Distribution of the displacement over a radius of the hollow sphere for several characteristic length distances $b$. Top: $b=0.5 \mathrm{~mm}$; Bottom-left: $b=1 \mathrm{~mm}$; bottom-right: $b=2 \mathrm{~mm}$. The computations were performed in the framework of linearized elasticity. Dashed lines: theory; dotted lines: GLPD numerical predictions. The numerical results agree very well with the analytical solution.

the GLPD model and loaded in tension can be viewed as a reference to assess future numerical implementations of the GLPD model into FE codes that differ from SYSTUS.

Another more complex numerical application is the problem of a pre-cracked axisymmetric specimen in tension, made of A508 Cl.3 steel, for which experimental results of fracture tests are available Rousselier and Mudry (1983). The mesh of this specimen is shown in Figure 2. Advantage is taken of symmetry about the horizontal mid-plane to mesh only the upper half of the structure. The semi-height and radius are $22.5 \mathrm{~mm}$ and $7.5 \mathrm{~mm}$ respectively. The shape of the central notch is triangular in a meridian plane; its half-opening angle and depth are $30^{\circ}$ and $2.5 \mathrm{~mm}$ respectively. A fatigue pre-crack of length $0.9 \mathrm{~mm}$ (invisible in the figure) originates from the notch root. Figure 3 shows the experimental and numerical load-displacement curves. The numerical results have been obtained using the GLPD model with $b=55 \mu \mathrm{m}$. The agreement is quite acceptable in view of the experimental errors.

The conclusion is that, although the computations using Enakoutsa and Leblond (2009)'s numerical scheme are quite time-consuming due to the increased number 


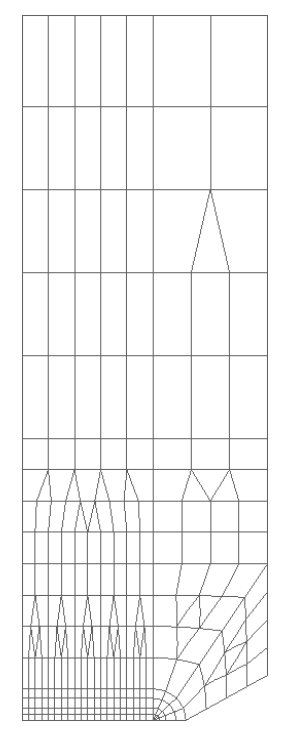

Figure 2: Mesh of the pre-cracked specimen

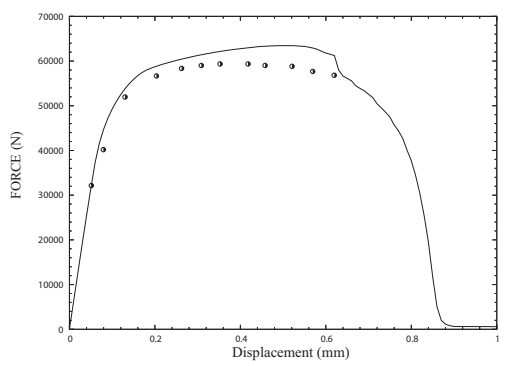

Figure 3: Comparison of experimental and numerical load-displacement curves of the pre-cracked specimen TA15. The agreement between the GLPD numerical predictions and the experiments is acceptable, in view of the experimental errors.

of nodal degrees of freedom, Enakoutsa and Leblond (2009)'s algorithm seems to be a promising tool to simulate the behavior of metallic materials undergoing ductile rupture, especially in the post-bifurcation regime of these materials.

\section{Aknowledgments}

This work was performed during the author's Ph.D. dissertation study at Pierre and Marie Curie University under Prof. Jean-Baptiste Leblond. His guidance is acknwoledged. 


\section{References}

Enakoutsa K., Leblond J.B. and Perrin G., 2007. "Numerical Implementation and Assessment of a Phenomenological Nonlocal Model of Ductile Rupture," Comput. Meth. Appl. Mech. Engng., 196, 1946-1957.

Enakoutsa K. and Leblond J.B., 2009. "Numerical Implementation and Assessment of a Phenomenological Nonlocal Model of Ductile Rupture," Comput. Meth. Appl. Mech. Engng., 196, 1946-1957.

Gologanu M., Leblond J.B., Perrin G. and Devaux J., 1997. Recent extensions of Gurson's model for porous ductile metals, in: Continuum Micromechanics, CISM Courses and Lectures 377, P. Suquet ed., Springer, pp. 61-130.

Gurson A.L. 1977. "Continuum Theory of Ductile Rupture by Void Nucleation and Growth: Part I - Yield Criteria and Flow Rules for Porous Ductile Media," ASME J. Engng. Materials Technol., 99, 2-15.

Pijaudier-Cabot, G. and Bazant, Z.P., 1987."Nonlocal Damage Theory", ASCE J. Engnrg. Mech., 113, 1512-1533.

Tvergaard, V., 1981. "Influence of voids on shear band instabilities under plane strain conditions," Int. J. Fracture, 17, 389-407.

Tvergaard, V. and Needleman, A., 1984. "Analysis of cup-cone fracture in a round tensile bar," Acta Metallurgica, 32, 157-169.

Rousselier, G. and Mudry, F., 1983. "Etude de la rupture ductile de l'acier faiblement allie en Mn-Ni-Mo pour cuves de reacteurs a eau ordinaire sous pression, approvisionne sous la forme d'une debouchure de tubulure. Resultats du programme experimental," EdF Centre des Renardieres Internal Report HT/PV D529 MAT/T43 (in French). 